\title{
Clinical, Pathological and Radio Logical Findings in the COVID-19 Positive Patients
}

\author{
Walaa Hussein Eltahir Elzuber ${ }^{1}$, Fatima Salih Ahmed Ezzeldein ${ }^{2}$, Mahmoud Galal Gheat ${ }^{1}$, \\ Ahmed Atef Soliman ${ }^{3}$, Angad Shaaban Diab ${ }^{1}$, Lubna Tagelsir Mohamed Khair ${ }^{4}$ \\ 1.Radiology specialist, King Khalid Hospital in Najran \\ 2.Radiology specialist, Alhyat national hospital, Gazan \\ 3.Radiology consultant, King Khalid Hospital in Najran \\ 4.Radiology resident, King Khalid Hospital in Najran
}

\begin{abstract}
Meanwhile, the outbreak of the COVID-19 pandemic, there is a severe need to learn related to its course, prognostic inflammatory markers, disease difficulties, and mechanical ventilation strategy. Clinically, three phases have been recognized founded on viral infection, pulmonary involvement with inflammation, and fibrosis. Although indispensable for therapeutic supervision, the pathophysiology of COVID-19 is badly unstated. Here, we methodically reviewed published case reports and case series in order to increase our comprehension related to KKH confirmed COVID-19 patient line list of kingdoms of Saudi Arabia, with the complete history of COVID-19 positive patients. Pathophysiology by constructing a timeline and connecting histopathological conclusions with clinical phases of COVID-19.
\end{abstract}

DOI: $10.7176 / \mathrm{JHMN} / 84-03$

Publication date: December $31^{\text {st }} 2020$

\section{Introduction}

Since late December 2019, an outbreak of a novel coronavirus disease was reported in Wuhan, China, that had harm 26 countries worldwide in the very first pandemic wave (Xie et al., 2020). In common, COVID 19 is a severe determined disease that caused $2 \%$ fatality rate. The acute disease inception might result in enormous alveolar harm and advanced respiratory failure. Until August 18, 2020, a total of 22,062,533 coronavirus cases had been reported throughout the world, with 777,668 deaths, and the number of patients that had recovered from the deadly disease are $14,805,177$. However, no pathology had been reported due to less available autopsy or biopsy. There are three successive phases of increasing severity in the COVID-19 (Wu, 2020). The initial stage of the infection with SARS-CoV-2 was identified. Flu - like symptoms may develop at this particular stage as a result of viral infection itself. The patient had developed viral pneumonia, which required hospitalization or even mechanical ventilation. Pulmonary and coagulopathic inflammation, which may continue successively but can often overlap, also categorize the second stage. Furthermore, enhanced rates are linked to the growth of ARDS (respiratory distress syndrome) and to an adverse path in inflammatory biomarkers such as $\mathrm{C}$ - reactive protein (CRP), and ferritin, as well as D- Dimer (Zhou F 2020). Finally, fibrosis is the third phase of the disease. Two respiratory phenotypes, the low (L) elastance and the large $(\mathrm{H})$ elastance, must be identified which are linked to those patients requiring mechanical ventilation. A higher pulmonary enema in H-type results in a higher pulmonary mass and less lung power. Furthermore, a notable proportion of cardiac output, when exchanging gases, perfuses unaerated lungs, which leads to high shunting from right to left. The supreme feature of type $\mathrm{H}$ is strong recruitment ability, indicating that the high volume of lung tissue that does not ventilate is used for gas exchanged by healthy and expiratory pressures.

As we know that, the symptoms appeared in covid19 patients with related to pulmonary infection, mild fever, cough, and lack of breath but it contains clinical symptoms as well such as vomiting, abdominal pain, and nausea. Many cases were reported about patients experience dry cough or abdominal dysfunction (GoldbergStein et al., 2020). The practice of pulmonary CT of patients who were diagnosed suspected COVID-19 has been encouraged. Numerous studies have been reported that the early detection of coronavirus disease through chest $\mathrm{CT}$ is could be more sensitive than (RT-PCR) reverse transcriptase polymerase chain reaction for the analysis of viral nucleic acid in patient (Xing et al., 2020), but RT-PCR is still preferable. In comparison with CT technique the ultrasound technique used for pulmonary infection is more convenient, cost effective and having very less ionizing radiation (Xing et al., 2020). However, another method to detect the positive SARS- CoV-2 RNA cases by (RT- PCR) reverse transcriptase polymerase chain reaction is the novel and standard method to diagnose the disease but it is time consuming method and observed the low supply of test kits cannot fulfil the demand of mass testing in suspected COVID-19 patients whereas, the SARS- CoV-2 RNA findings may not be reliable as showed deceptively negative results due to handling errors or inadequate samples (Xie et al., 2020). That's why the demand of radiological examination is high, especially lung CT because it is a non- invasive, advantageous and quick method to investigate the early and late pathological changes as well as the status of severity (Xie et al., 2020). Although, bilateral (GGOs) ground glass opacities or consolidation on pulmonary/chest CT is the 
most suitable way to diagnose the positive Covid 19 disease as indicated by earlier radiographical studies. Another novel findings were reported by (Goldberg-Stein et al., 2020) in which they mentioned about abdominopelvic CT with lung base appearance were suggested by radiologist in COVID-19 pneumonia cases. Although, some patients have abdominal dysfunction symptoms while others don't, and this mechanism is poorly investigated till yet.

Instead, the L - type ensurs because, due to damage of the infusion guideline, hypoxic vasoconstriction and the capacity of the lungs to function with the unaerated lung tissue is truncated, the usual lung V/ Q ratio is diminished (Gattinoni, 2020). Similar pathophysiological mechanisms should therefore significantly fluctuate between low and high respiratory phänotypes requiring different treatments with different consequences. There is poor understanding of the pathological mechanism that drives these two respiratory phenotypes, while in February 2020 first advice has been released on the current and apparently positive COVID-19 patients (Hanley, 2020). There have been an increasing number of biopsies and autopsies. Nonetheless, in order for us to employ closely flapped, anti - inflammatory, or antifibrotic antipersonnel, our awareness of the exact nature of immunological resistance in various organ plans as a response to viral infection, as well as of specific tissue reactions, is predominantly incomplete, yet necessary.

In order to identify a timeline for associating histopathological reactions with different COVID-19 clinical phases, an analytically study is required for the current cases details and the case series reporting abnormal symptoms to COVID-19 patients.

The aim of this study was to understand the pathophysiological approaches developed in many phases of the disease to improve the implementation of beleaguered therapies in King Khalid Hospital in Najran, South Saudi Arabia.

\section{Methodology of the study Study Design, setting and period}

In this a cross sectional hospital based study, conducted in King Khalid Hospital in Najran, South Saudi Arabia, during the period from 1 March to 31 August, 2020.

\section{Data Collection}

The study surveys about 890 coronavirus patients having different nationalities. The patients belonged to the different countries and were residing in Saudi Arabia.

Data was collected by personal interview of the patients and using a predesigned questionnaire, it includes questions about their information which includes their nationality, age, mobile number, ID card number, MRN, and HESN patient ID, LAB requisition ID, symptoms onset date, region, city and case status, etc.

Afterward, the questions about their travel history, and where were they travel including their entry in the kingdom of Saudi Arabia, also the inquiry about the contact with any confirmed case of corona virus has been conducted, even the name of confirmed cases, the date of contact with them if there is any, including the inquiry with the patient that the patient visits any other facility has been entertained.

\section{Statistical analysis}

In this study, there are two types of data analysis methods, i.e., Quantitative and Qualitative data analysis. This study focused on the quantitative data analysis technique due to the data collected from the survey.

\section{Results and findings}

The number of patients was 890, including Afghanistan, Sudan, Arab states, Yemen, and Pakistan as well.

The findings in this study are a result of the collected questionnaire. Empirical assessment is performed considering the evaluation of the KKH confirmed COVID-19 patients. Results are based on the quantitative approach. The responses of the questionnaires are summarized followed by demographic and evocative analysis to reach the tangible conclusions.

The survey document comprised of the basic information of the COVID-19 positive patients, as shown in figure 1 , that is presenting the nationality of the patients. $57 \%$ of the patients belongs to the kingdom itself and the nationals of Saudi Arabia. Whereas, 9\% of the total patients belongs to Egypt and they are Egyptian. While $7 \%$ of the patients belongs to Bangladesh, $10 \%$ patients are Yemeni, $7 \%$ patients belong to India, $2 \%$ of the total patients are Pakistani nationals, $1 \%$ belong to Syria, 3\% are unknown, while Qatar, Nepal, Indonesia, Somalia, are at $0 \%$ respectively.

Regarding radiological examination, we observed positive lung US findings in suspected covid19 patients $(n=28)(50 \%$ ratio of men and women) with an average age of $(59.8 \pm 18.3)$. Findings revealed that, the pleural line of lung was thickened in most cases with longer period of disease as compared to pulmonary consolidations were mostly found in severe and critical cases than in moderate ones. The findings ratio are as follows (B-lines found in $100.0 \%, 28 / 28$ patients, consolidation found in $67.9 \%, 19 / 28$ patients, and a thickened pleural line was 
observed in $60.7 \%, 17 / 28$ patients). In another survey, we observed CT images which was pure or mixed (GGOs) ground glass opacities was not significantly different in suspected COVID-19 patients and SARS- CoV-2negative patients $(75.0 \%$ vs $62.5 \%$ with $\mathrm{p}=0.687)$, but more commonly found in COVID-19 patients. There were no significant differences in the presence of pulmonary inflammatory symptoms appeared in both cases. However, 54\% COVID 19 patients who were positive and having abdominal pain which is considered as the common sign of disease. While the patients having abdominopelvic CT were age between 64 years in which 91 were females. Although, 80 patients $(57 \%)$ had positive abdominopelvic findings.

All the patients are belonging to the region of Najran as shown in the figure 2 .

Case status is showing that all the suspected number of COVID-19 patients are confirmed cases as showing in figure 3 that 890 out of 890 patients are confirmed affected with corona virus.

The survey has inquired about the travel history of the patients and it tells that $3 \%$ out of 890 patients have answered positively whereas, $56 \%$ out of 890 answered negatively while the other remain unanswered. As showing in figure 4.

Travel history of the patients shows that $88 \%$ of the patients didn't travel anywhere, while $6 \%$ travel to Saudi Arabia, 4\% leave it blank, whereas, $1 \%$ travel to Yemeni border and Yemen respectively. As shown in figure 5.

The patients have been inquired about the contact history with any COVID-19 positive patients before they are infected $26 \%$ of the patients replied in negative whereas, $4 \%$ of the patients replied in positive while rest of $70 \%$ patients remains unanswered, as figure 6 is showing.

The patients have been inquired about visiting any other facility the $90 \%$ of the patients have replied with no and $4 \%$ with yes while the 5\% with N/A and the remaining $1 \%$ left it blank. As shown in figure 7 .

The patients answer the questions of specifying the names of the facility if any with $43 \%$ cardiac unit, $11 \%$ left it blank, $3 \%$ pf the patients reply with $\mathrm{KKH}, 41 \%$ have replied with no while $1 \%$ replied with NNGH the remaining o \% with SGH, yes, Yadama, habona, PHC- moratha, respectively as shown in Figure 8.

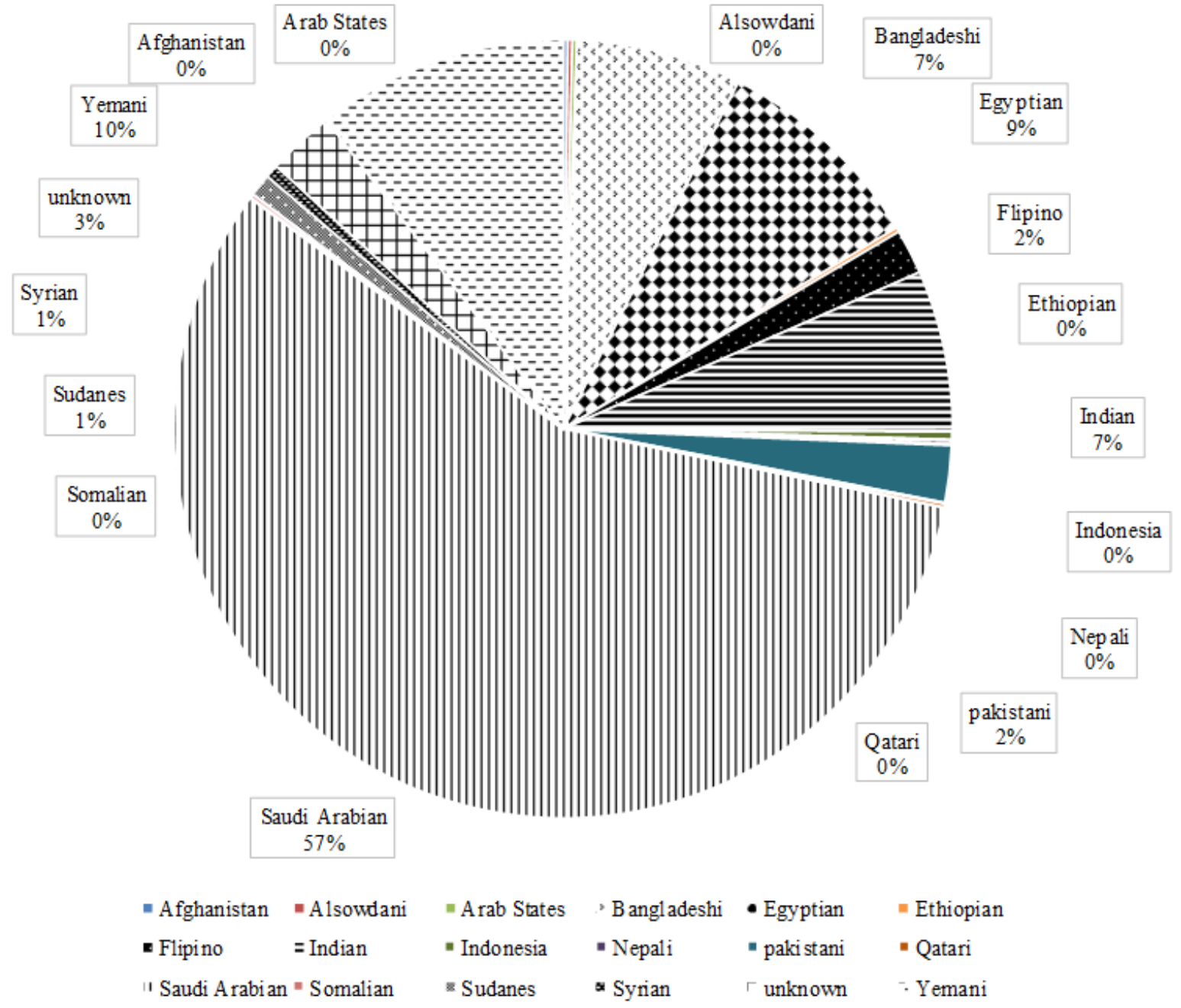

Fig. 1 Nationality the nationality COVID-19 positive patients 


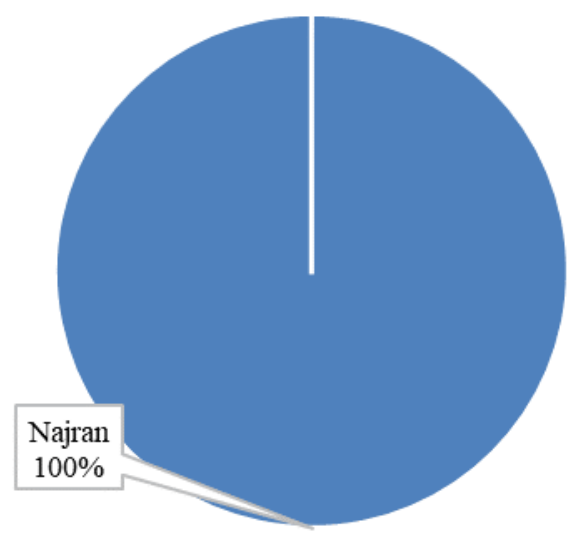

- Najran

Fig.2 Region of the patients

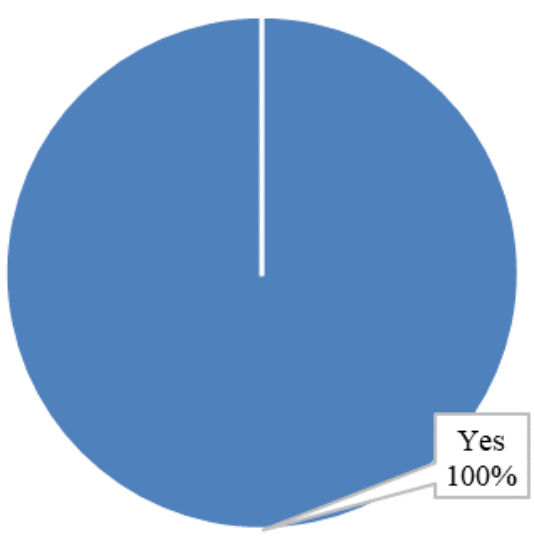

- Yes

Fig. 3 Case Status of the patients

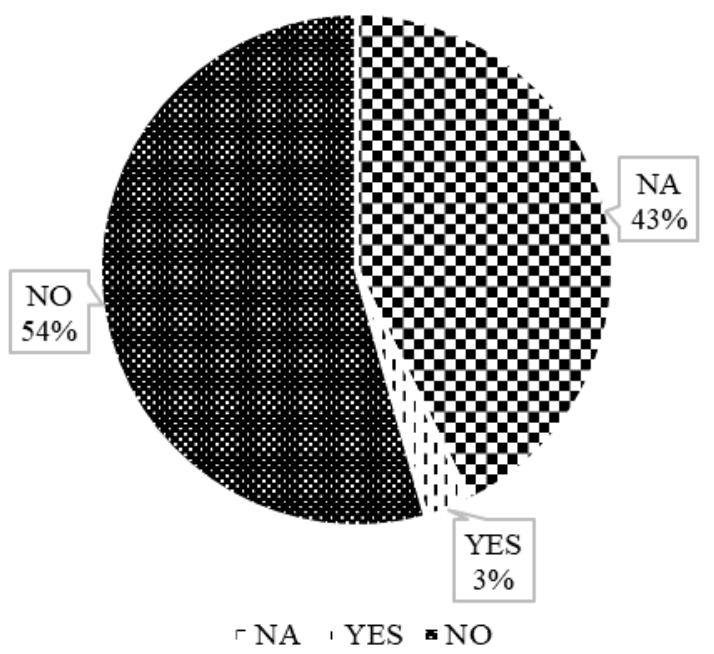

Fig.4 Is there a travel history abroad recently? 


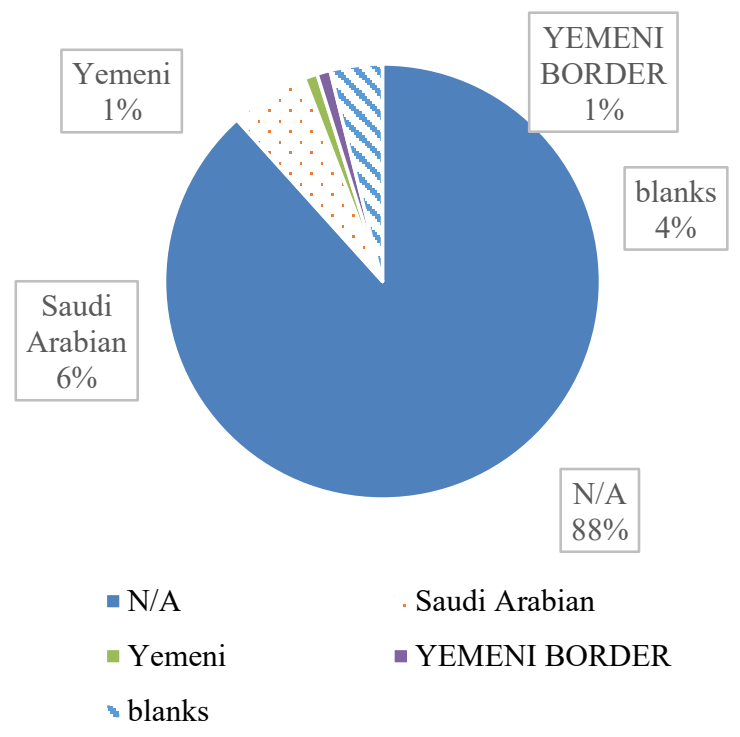

Fig.5: the region where the patients travel to it

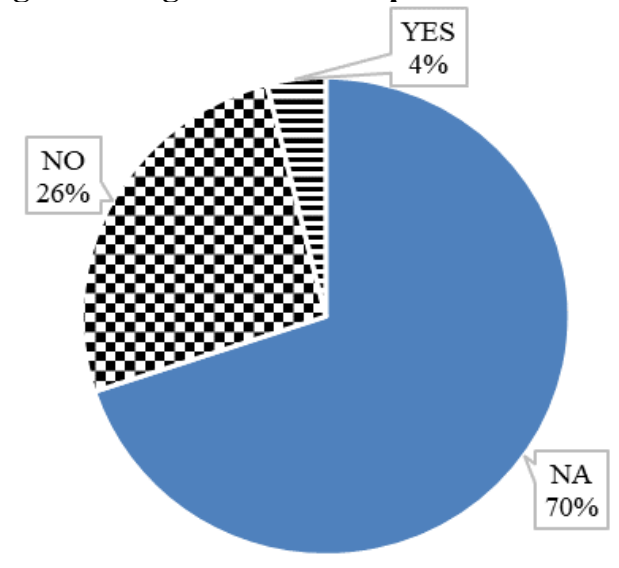

- NA YNO - YES

Fig (6): Is there contact history with a confirmed case?

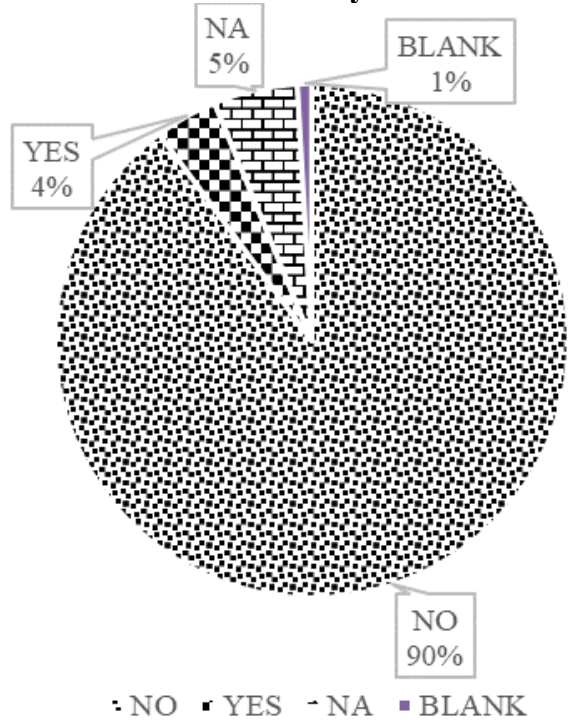

Fig.7 Did the patient visit any other facility 


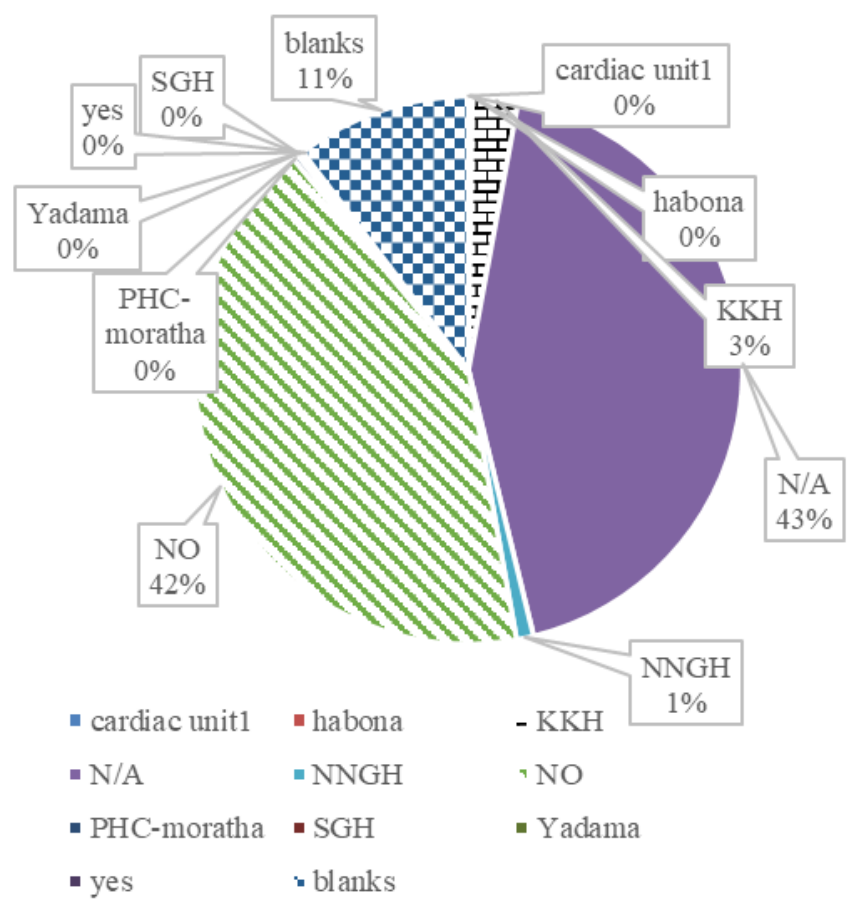

Fig.8 specify the name of the facility, if any

\section{Discussion}

The construction among these pathological discoveries and the scientific course of COVID-19 designates the possibility that the pathogenesis trails a consecutive pattern (Siddiqi HK 2020)

In the initial stage of viral infection, respirational epithelial cells are infested; therefore, epithelial vicissitudes preponderate with indication of viral activity. Succeeding viral permission is contracted in patients with unembellished disease or underlying risk issues. The existence of epithelial lung injury with hyperplasia and atypia of pneumocytes and multinucleation, probably representative of viral cytopathic effects, even in advanced stages of COVID-19, provisions this finding (Liu Y 2020).

In this initial epithelial stage, if histopathological modifications are slight and only a incomplete amount of lung tissue is pretentious, low-type elastance lung changes are more mutual. In the subordinate stage of the illness, advanced respiratory abnormalities can grow, leading to high lung elastance respiratory phenotype. This development from truncated to high elastance can be accredited to numerous features, as well as: self-inflicted lung injury by the patient. (C. D. Gattinoni L 2020) ventilator-induced lung injury microvascular injury; hypercoagulability associated with activation of the complement pathway; (Magro C 2020) activation of the innate immune activation, with triggering of a proinflammatory response, including the cytokines IL-1 $\beta$, IL6, and TNF- $\alpha$; and an adaptive T cell-mediated immune response (Felsenstein S 2020)

Prominently, thrombotic difficulties frequently progress in this phase of the disease. (Klok FA 2020). However, several hyperinflammatory individuals with secondary hemophagocytic lymph histiocytosis, which has hypertyrosinemia, are also seen at the very early stage of the disease (Mehta P 2020). This could have happened in those who had the most chronic lung injury history at the early stages of the disease. Unfortunately, the D-dimer level was not correlated with the presence or extent of vascular changes in this study, as only small events contained information on D-dimer rates. Fibrosis also occurs in patients approaching the final stage of the disease with a worsening inflammatory response (Chen JY 2020).

Our analysis showed a significant difference in the number of histopathological findings in the pulmonic of COVID-19 with standard ARDS and other types of viral pneumonitis, namely acute interstitial pneumonia with enema and DAD with different levels of organisation, usually found in ARDS. (Thille AW 2013).Influenza pneumonitis, the past of histopathology also involves capillary and small vessel thrombosis, interstitial oedema, interstitial neutrophil and lymphoblastic inflammatory diseases (NAID) in addition to brominates and bronchiolitis, the development of hyalines membranes, varying degrees of intra - alveolar edemaand/or hemo and acute DAD (von der Thusen J 2020). In addition, patients experience no occultation of airway debris owing to the airway denudation as well as inflammatory cell infiltrations, haemorrhage, alveology and the formation of the hyaline membrane, common clinical characteristics from the exudative stage of DAD, for example in the Mideastern Respiratory Syndrome and former severe acute respiratory syndrome induced by SARS-CoV (Gralinski LE 2020). 
The histopathological description of pneumonitis in COVID-19 may tend to be relativistic ally dependable epithelial, vascular and fibrotic lung injury trends. Through examination of those patterns in different stage diseases with respect to symptoms, we have noticed a comparatively clear timeline. As an example, epithelial, vascular and fibrotic forms of lung injury appear to include the histopathologic description of COVID-19-related pneumonitis. They also built a relatively clear time line by analyzing these patterns of patients with various phases of the disease prior to the onset in symptoms. In addition, epithelial changes - like DAD, denudation and receptive atypical pneumocyte - were observed throughout all stages of illness (Ackermann M 2020).

Hyaline membrane development, fibrin exudates, epithelial disruption and diffuse pneumocyte hyperplasia of type II, all of which are symptoms of the DAD, are the major pathologic findings in the lungs. Moderate thick of the alveolar walls becomes evident in some instances, indicating a more advanced stage (Tian S 2020). Even though, options of treating severe cases of COVID-19 are needed urgently, these treatments are currently not available. Though attempting to inhibit the virus, replication may be a viable option in initial stages of the disease, the method present itself as less effective in the later stage, in that inflammatory phenotype dominates the clinical picture. (Grein J 2020).

Therefore, choices concerning the use and timing of either antiviral agents or anti-inflammatory drugs may lead to a problematic dilemma in the non-appearance of thorough knowledge regarding the underlying pathophysiology. (Wang Y 2020). Decisions on the use and timing of antiviral agents or anti - inflammatory drugs can therefore contribute to a very complicated problem in the absence of detailed understanding of the pathophysiology. In a significant number of affected countries, autopsies have been carried out by pathologist, with or without peer review (Grein J 2020).

It is noted that, previously, the US (ultrasound) for lung imaging had been widely used for Ebola disease (also known as haemorrhagic fever) containing patient found in the isolation centre to gain information about disease (Xing et al., 2020). Currently, at the time of Covid 19 pandemic the usage of US over CT has been gain momentum in medical management because it is reliable and cost-effective technology. However, the widely use pulmonary US imaging were B-lines. B-lines can picture as many vertical echogenic resonances, extending from the lung's surface. But, B-lines has specific function and some lacking such as it cannot be detecting causes of diseases, duration and their severity such as acute respiratory distress syndrome, cardiogenic edema, pulmonary fibrosis, and viral pneumonia etc. on the other hand, the specificity of chest ultrasound regarding covid 19 existence in patients is predictable to be very low at initial appearance. Although, B-lines was preferable in Covid patients and showed thickened pleural line was found in a multiple case with higher duration of disease which is probably reflecting the pulmonary fibrosis. Although, after the initial symptoms appear the US findings showed a thick pleural line with longer time intervals was observed in Covid patients, while, the severe and critical stages in lungs can be easily detected by consolidation in which we can visualized higher inflammatory cellular exudate with found high rate of low air flow. While, the thickening of interlobular and intralobular septa can be observed during chest CT, but early diagnosis of COVID-19 through chest CT is very effective to tackle the disease. Furthermore, the use of chest US imaging enabled to predict and analysed the disease progression, as well as the pathophysiology and history of COVID-19 (Xing et al., 2020). Similarly, chest CT is more complex than Xray (CXR) on different stages of diagnosis, initial and response examination of COVID-19 as reported by (Xie et al., 2020). It is noted that, CXR having no abnormal results on early stage of COVID-19, while CT evaluate the disease before the symptoms appear. On the other hand, CT imaging is considered as first line of source to detect the disease more efficiently in china. Various studies have been reported about CT findings especially in COVID-19 cases which were comprised on pulmonary inflammations such as pulmonary parenchymal, pure or mixed GGOs in the lungs margin, interlobular septal and intralobular interstitial thickening, consolidation, and paving patterns (Xie et al., 2020). Whereas, similar findings were also observed in CXR. Findings on abdominopelvic CT in COVID-19patients is consider an important study because abdominal discomfort is a common sign in covid patients whereas it is also recorded that mostly patients do not experience this sign despite positive. Nearly $57 \%$ of patients have positive abdominopelvic discoveries on CT and half were not probably $64 \%$ with either middle or typical COVID- 19 pulmonary findings at the lung bases (GoldbergStein et al., 2020).

\section{Conclusion}

The study aimed at identifying therapeutic processes, physiological and radiological outcomes and possible COVID-19 treatment choices. Several patients reported cough, diarrhea, shallow breathing and myalgia, although few patients were able to develop pneumonia and ARDS. The radiological and pathological observations are widely observed, as are consensus - based opaqueness of ground vessels in lungs periphery, partnerships, needless paving patterns, indications of the air bronchograms, diffuse alveolar injury, bronchial fibrinity exudates, hyalin membranes production, inflammatory cells penetration and alveolar metaplastic alteration. However, the work of antivirals such as remdesivir and lopinavir, azithromycin, hydroxychloroquine and passive vaccine such as convalascent plasma is being investigated. There are no conclusive treatments 
available. The emphasis is at present on disease dissuasion through working social disaffection until a vaccine is created. More work on the development of vaccines and training of the health community and the public are expected extensively. In future, to prevent such injuries, drastic procedures are required.

The study suggested that the three stages of COVID-19 should be followed: it should therefore be noted that these phases can occur successively or instantly. Satisfactory treatment based on the underlying pathophysiological process should be offered from a therapeutic perspective. When virus infection is part of the pathogenic concept antiviral drugs are more effective. During the vascular stage directions are suggested against harmful, anticoagulant and/or anti-complement agent. Finally, the precise pathophysiological status of an individual cannot be established through tissue biopsy or by clinical testing if the fibrosis is producing biologic and/or small antifibrotic molecule compounds including those at the experimental stage.

The study suggest that the total number of patients belongs to different nationalities were residing in the kingdom of Saudi Arabia, tested positive for corona virus. The study has gathered their basic information along with their travel history and the place of where they travel to. Including the contact history with COVID-19 patient, and lastly whether they had visited any other facility. Results are calculated on the basis of information that has been provided.

In conclusion regarding radiological finding in Covid 19, the pulmonary abnormalities can be analysed by US (ultrasound) techniques with, B-lines, thick pleural line, and consolidation which has been using for COVID19 patients and it is very compatible and cost free. Although, after the initial symptoms appear the US findings showed a thick pleural line with longer time intervals was observed in Covid patients, while, the severe and critical stages in lungs can be easily detected by consolidation. In addition, pulmonary US help to evaluate to identify the severe and critically ill patients affected by Covid 19. forms of the disease. The US imaging can be repeatable to check the severity of disease as well as the effectiveness of therapeutic treatment in critically ill patients. Although, another method in lung CT is GGOs (ground glass opacities) can help to identify suspected but negative patients with COVID-19 but CT cannot replace by reverse transcriptase polymerase chain reaction method. In COVID 19 the abdominal discomfort is the common sign of the presence of disease. Even though, Abdominopelvic CT were performed on positive covid patients and found positive results in $57 \%$ patients while half of the patients had no positive finding on abdominopelvic CT.

\section{References}

1. Ackermann M, Verleden SE, Kuehnel M, Haverich A, Welte T, Laenger F, et al. 2020. "Pulmonary vascular endothelialitis, thrombosis, and angiogenesis in Covid-19." N Engl J Med.

2. Chen JY, Qiao K, Liu F, Wu B, Xu X, Jiao GQ, et al. 2020. " Lung transplantation as therapeutic option in acute respiratory distress syndrome for COVID-19-related pulmonary fibrosis." Chin Med J (Engl).

3. 2020. "Collection and Submission of Postmortem Specimens from Deceased Persons with Known or Suspected COVID-19 (Interim Guidance) " https://www.cdc.gov/coronavirus/2019-ncov/hcp/guidancepostmortem-specimens.html.

4. Felsenstein S, Herbert JA, McNamara PS, Hedrich CM. 2020. "COVID-19: immunology and treatment options." Clin Immunol.

5. Gattinoni L, Chiumello D. 2020. "COVID-19 pneumonia: different respiratory treatments for different phenotypes." Intensive Care Med.

6. Gattinoni L, Chiumello D, Caironi P, Busana M, Romitti F, Brazzi L, et al. 2020. "COVID-19 pneumonia: different respiratory treatments for different phenotypes?" Intensive Care Med.

7. Gralinski LE, Baric RS. 2020. "Molecular pathology of emerging coronavirus infections." J Pathol.

8. Grein J, Ohmagari N, Shin D, Diaz G, Asperges E, Castagna A, et al. 2020. "Compassionate use of remdesivir for patients with severe Covid-19." N Engl J Med.

9. Hanley B, Lucas SB, Youd E, Swift B, Osborn M. 2020. "Autopsy in suspected COVID-19 cases. ." J Clin Pathol.

10. Klok FA, Kruip M, van der Meer NJM, Arbous MS, Gommers DAMPJ, Kant KM, et al. 2020. " Incidence of thrombotic complications in critically ill ICU patients with COVID-19." Thromb Res 145-7.

11. Liu Y, Yan LM, Wan L, Xiang TX, Le A, Liu JM, et al. 2020. " Viral dynamics in mild and severe cases of COVID-19." Lancet Infect Dis 656-7.

12. Magro C, Mulvey JJ, Berlin D, Nuovo G, Salvatore S, Harp J, et al. 2020. "Complement associated microvascular injury and thrombosis in the pathogenesis of severe COVID-19 infection." a report of five cases. Transl Res.

13. Mehta P, McAuley DF, Brown M, Sanchez E, Tattersall RS, Manson JJ, et al. 2020. "COVID-19: consider cytokine storm syndromes and immunosuppression." Lancet.

14. Siddiqi HK, Mehra MR. 2020. "COVID-19 illness in native and immunosuppressed states: a clinicaltherapeutic staging proposal." J Heart Lung Transpl. 405-7.

15. Thille AW, Esteban A, Fernandez-Segoviano P, Rodriguez JM, Aramburu JA, Peñuelas O, et al. 2013. 
"Comparison of the Berlin definition for acute respiratory distress syndrome with autopsy." Am J Respir Crit Care Med. 761-7.

16. Tian S, Hu W, Niu L, Liu H, Xu H, Xiao SY. 2020. "Pulmonary pathology of early-phase 2019 novel coronavirus (COVID-19) pneumonia in two patients with lung cancer." J Thorac Oncol.

17. von der Thusen J, van der Eerden M. 2020. "Histopathology and genetic susceptibility in COVID-19 pneumonia." Eur J Clin Invest.

18. Wang Y, Zhang D, Du G, Du R, Zhao J, Jin Y, et al. 2020. "Remdesivir in adults with severe COVID-19: a randomised, double-blind, placebo-controlled, multicentre trial. ." Lancet.

19. Wu C, Chen X, Cai Y, Xia J, Zhou X, Xu S, et al. 2020. " Risk factors associated with acute respiratory distress syndrome and death in patients with coronavirus disease 2019 pneumonia in Wuhan, China." JAMA Intern Med.

20. Zhou F, Yu T, Du R, Fan G, Liu Y, Liu Z, et al. 2020. "Clinical course and risk factors for mortality of adult inpatients with COVID-19 in Wuhan, China: a retrospective cohort study." Lancet 1054-62.

21. Xing, C., Li, Q., Du, H., Kang, W., Lian, J., \& Yuan, L. (2020). Lung ultrasound findings in patients with COVID-19 pneumonia. Critical Care, 24(1), 1-5. https://doi.org/10.1186/s13054-020-02876-9

22. Xie et al. (2020). Chest CT- based differential diagnosis of 28 patients with suspected corona virus disease 2019 ( COVID-19). May.

23. Goldberg-Stein, S., Fink, A., Paroder, V., Kobi, M., Yee, J., \& Chernyak, V. (2020). Abdominopelvic CT findings in patients with novel coronavirus disease 2019 (COVID-19). Abdominal Radiology, 2019(0123456789). https://doi.org/10.1007/s00261-020-02669-2 